\title{
Optical control of epigenetics
}

Reis et al. present chemo-optical modulation of epigenetically regulated transcription (COMET), a new conceptual approach that harnesses the strengths of small-molecule inhibitors and optogenetic techniques to control the epigenome using light.

Pharmacological small molecules and optogenetics are powerful tools to interrogate the epigenetic regulatory landscape; however, they suffer from insufficient spatiotemporal resolution, are limited to threshold-triggered responses or require genetic manipulation of the target cell.

The group designed hybrid histone deacetylase (HDAC) inhibitors that contain azobenzene, which is capable of photoisomerization upon exposure to blue light. This enabled the team to modulate the affinity for the HDAC by controlling exposure to light. Combining structural modelling, in vitro assays and cell biology work, the authors showed that upon light activation the modified HDAC inhibitors bind to their targets and prevent the modification of the histone residue H3K9. The team performed high-throughput multiplexed mRNA expression profiling on cells that were treated with the inhibitors and then exposed to light. By comparing treated with control cells kept in the dark or lacking inhibitor treatment, the authors identified a set of 120 core genes that were differentially expressed in treated cells upon exposure to light. Network analysis revealed that core genes were associated with cell cycle regulation and mitochondrial function.

To manipulate epigenetics without requiring genetic manipulation of a target cell could allow researchers to address complex in vivo biological processes in unaltered cells or even whole organisms.

Ross Cloney, Associate Editor, Nature Communications

ORIGINAL ARTICLE Reis, S. A. et al. Lightcontrolled modulation of gene expression by chemical optoepigenetic probes. Nat. Chem. Biol. http://dx.doi.org/10.1038/nchembio.2042 (2016)

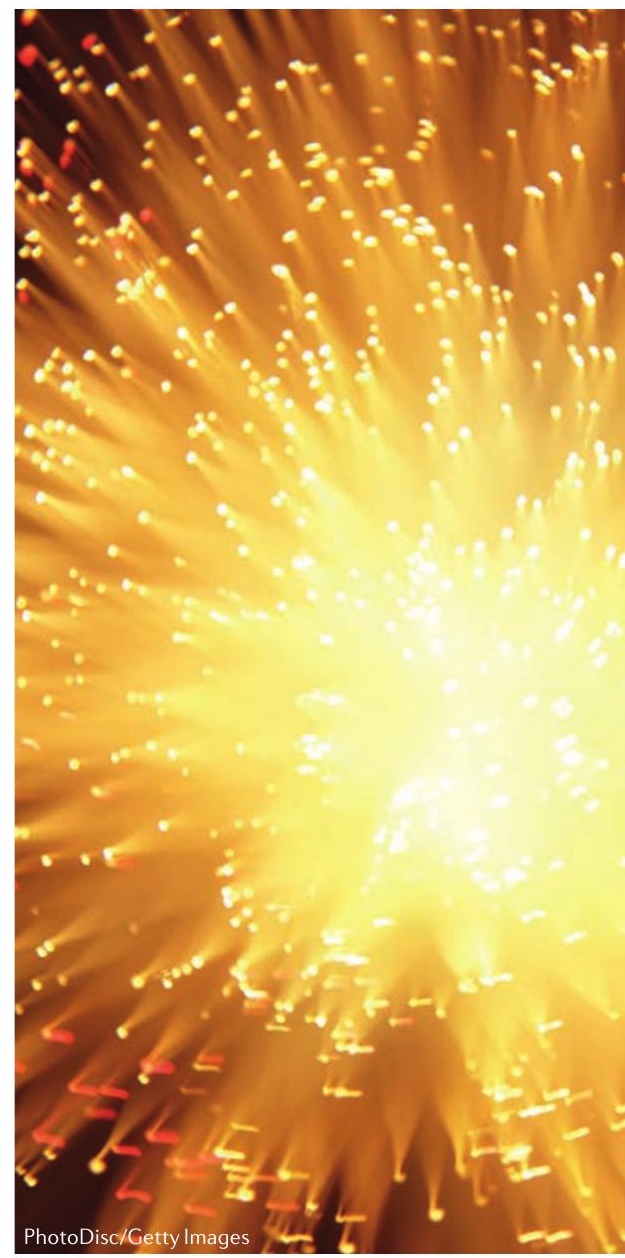

